\title{
December 2013 Imaging Case of the Month
}

\author{
Michael B. Gotway, MD \\ Department of Radiology \\ Mayo Clinic Arizona \\ Scottsdale, AZ
}

Clinical History

A 30-year-old man complained of intermittent shortness of breath and recurrent respiratory tract infections. Frontal chest radiography (Figure 1) was performed.

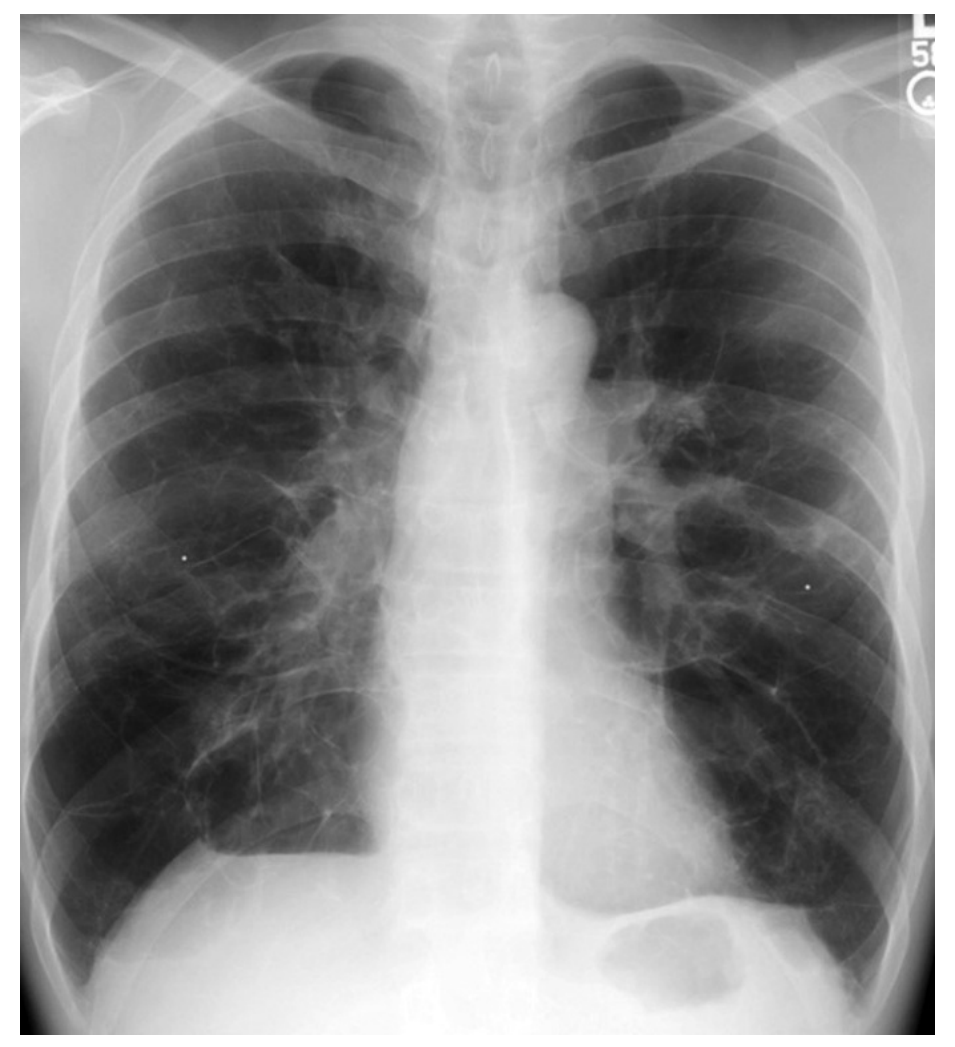

Figure 1. Frontal chest radiograph.

Which of the following statements regarding the chest radiograph is most accurate?

1. The chest radiograph mediastinal lymphadenopathy

2. The chest radiograph shows basal predominant linear opacities suggesting fibrosis

3. The chest radiograph shows large lung volumes with faint, thin-walled cystic lesions bilaterally

4. The chest radiograph shows multifocal ground-glass opacity and consolidation associated with linear and reticular abnormalities

5. The chest radiograph shows multiple nodules 


\section{Correct! \\ 3. The chest radiograph shows large lung volumes with faint, thin-walled cystic lesions bilaterally}

The chest radiograph shows large lung volumes, which argues strongly against a fibrosing lung process. Patchy perihilar linear and reticular opacities are present, but no infiltrative findings, such as ground-glass opacity and consolidation, are evident. The ascending aorta is mildly tortuous, and is the cause of the right mediastinal contour, but no mediastinal or hilar lymph node enlargement is evident. There are no pulmonary nodules. In addition to the large lung volumes, curvilinear opacities are present, creating the appearance of faintly seen cysts, best appreciated in the left perihilar region. In this area, a small air-fluid level may be present as well (Figure 2).

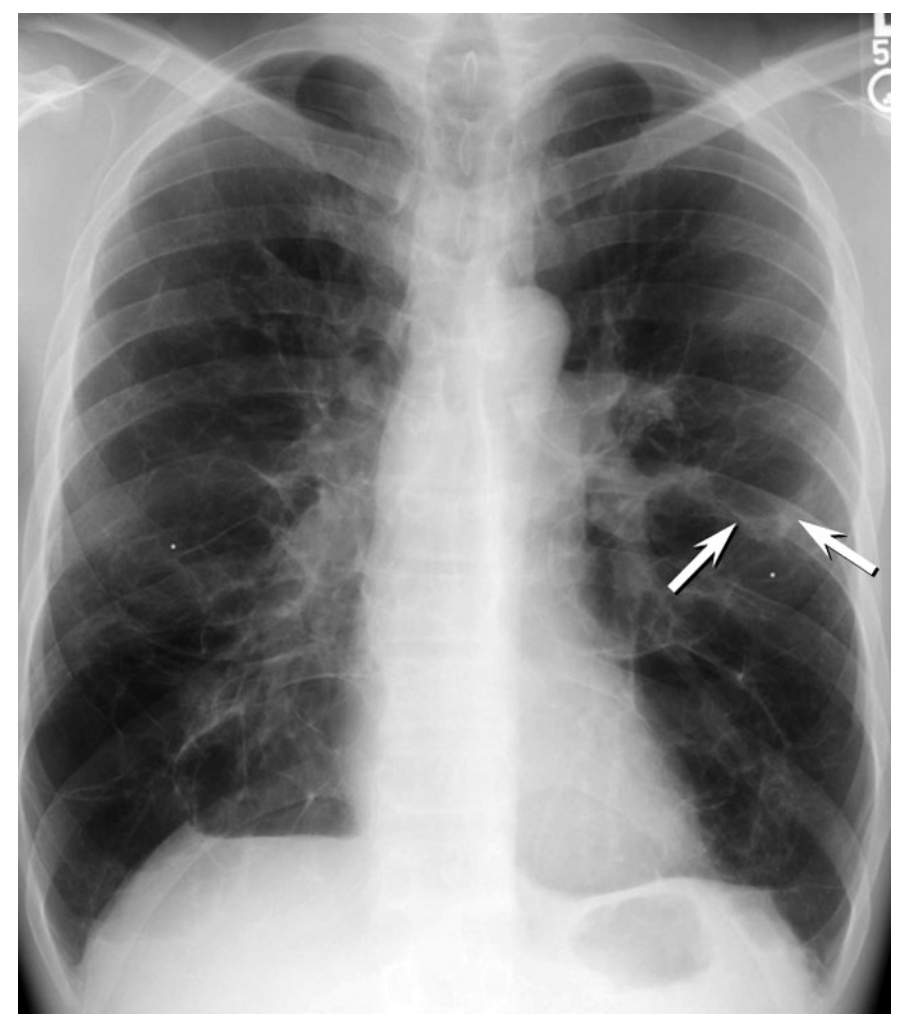

Figure 2. Frontal chest radiography shows large lung volumes associated with bilateral perihilar linear and curvilinear opacities that create the faint appearance of thin-walled

cysts. A small, low air-fluid level (arrows) may be present in the left perihilar region.

Which of the following is the most appropriate consideration among the differential diagnostic possibilities for the appearance of the patient's chest radiograph?

1. Aspirated foreign body

2. Bronchiectasis

3. Disseminated infection

4. Lymphoma

5. Usual interstitial pneumonia / idiopathic pulmonary fibrosis 


\section{Correct! \\ 2. Bronchiectasis}

The large lung volumes and lack of basal predominant, subpleural linear and reticular opacities excludes usual interstitial pneumonia / idiopathic pulmonary fibrosis as a diagnostic consideration. Disseminated infection typically presents on chest radiography as multiple variably-sized nodules, sometimes "miliary" in appearance, possibly with other infiltrative abnormalities, such as consolidation, none of which are seen on this chest radiograph. An aspirated foreign body may be radiographically occult, or may appear as focal increased lucency within a portion of a lung or affecting the entire lung, resulting from endobronchial obstruction with air trapping distal to the point of obstruction. Occasionally the foreign body itself may be visible, if it is radiopaque. Lymphoma manifestations within the thorax are protean, but commonly include mediastinal lymph node enlargement, possibly hilar lymphadenopathy as well, or single or multiple pulmonary nodules or masses, with or without pleural effusion- none of these findings are evident. The large lung volumes and curvilinear opacities suggesting the possibility of pulmonary cysts raises the possibility of various causes of cystic lung disease, including bronchiectasis.

The patient underwent thoracic CT (Figure 3) for further characterization of the chest radiographic findings.

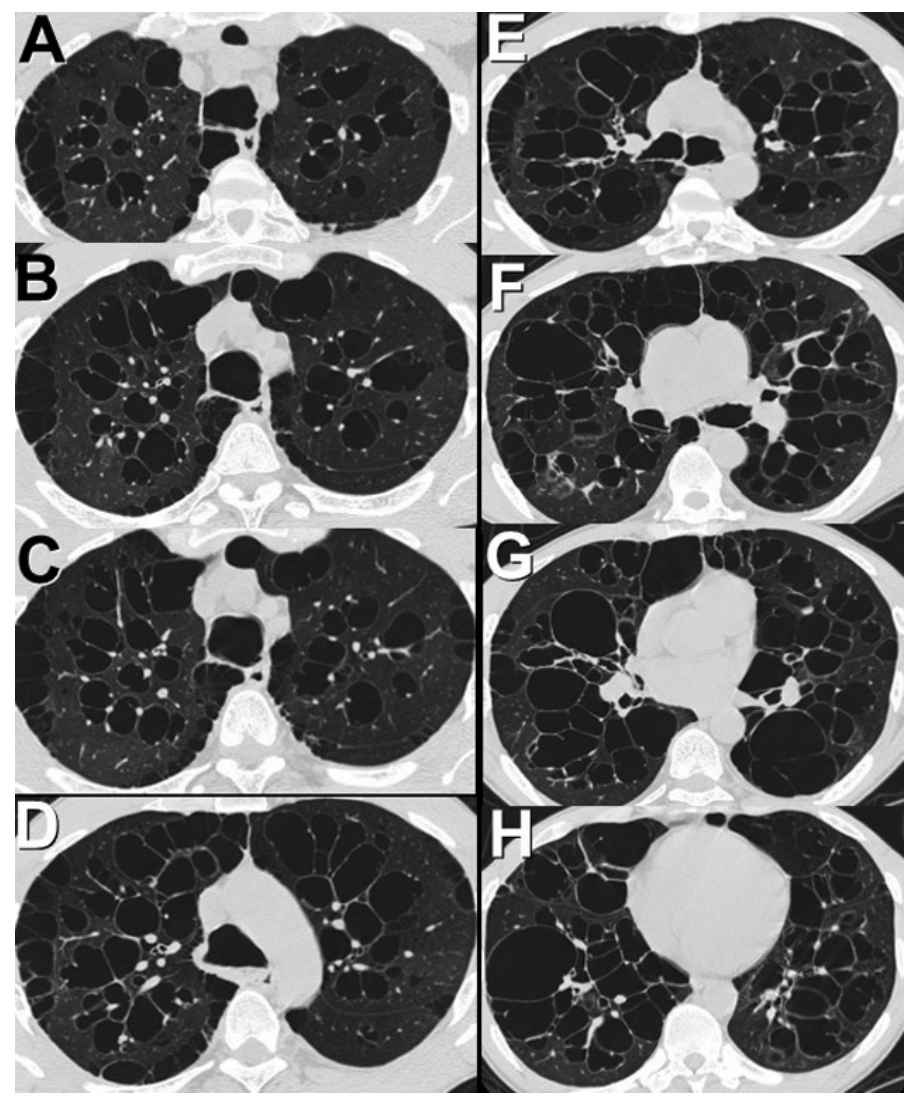

Figure 3. Representative axial thoracic CT images displayed in lung windows. 
Which of the following statements regarding this CT examination is most accurate?

1. The thoracic CT shows extensive centrilobular emphysema

2. The thoracic CT shows multifocal areas of lobular low attenuation suggesting air trapping form small airway obstruction

3. The thoracic CT shows multifocal thick-walled cavities suggesting neoplasia

4. The thoracic CT shows numerous thin-walled cysts consistent with lymphocytic interstitial pneumonia

5. The thoracic CT shows numerous thin-walled cysts representing bronchiectasis 


\section{Correct! \\ 5. The thoracic CT shows numerous thin-walled cysts representing bronchiectasis}

The thoracic CT shows numerous thin-walled cystic structures; these account for the curvilinear opacities detected at chest radiography. Once recognized, the differential considerations for cystic pulmonary lesions include the numerous causes of cystic pulmonary diseases 9such as Langerhans cell histiocytosis, lymphangioleiomyomatosis, lymphocytic interstitial pneumonia, hypersensitivity pneumonitis, and Birt-Hogg-Dubé syndrome, among others), emphysema, air trapping, cystic pulmonary metastases, and bronchiectasis. Centrilobular emphysema can be distinguished from cystic lung disorders and bronchiectasis by the presence of a "dot" within the center of the low attenuation area, which represents the centrilobular artery (Figure 4).

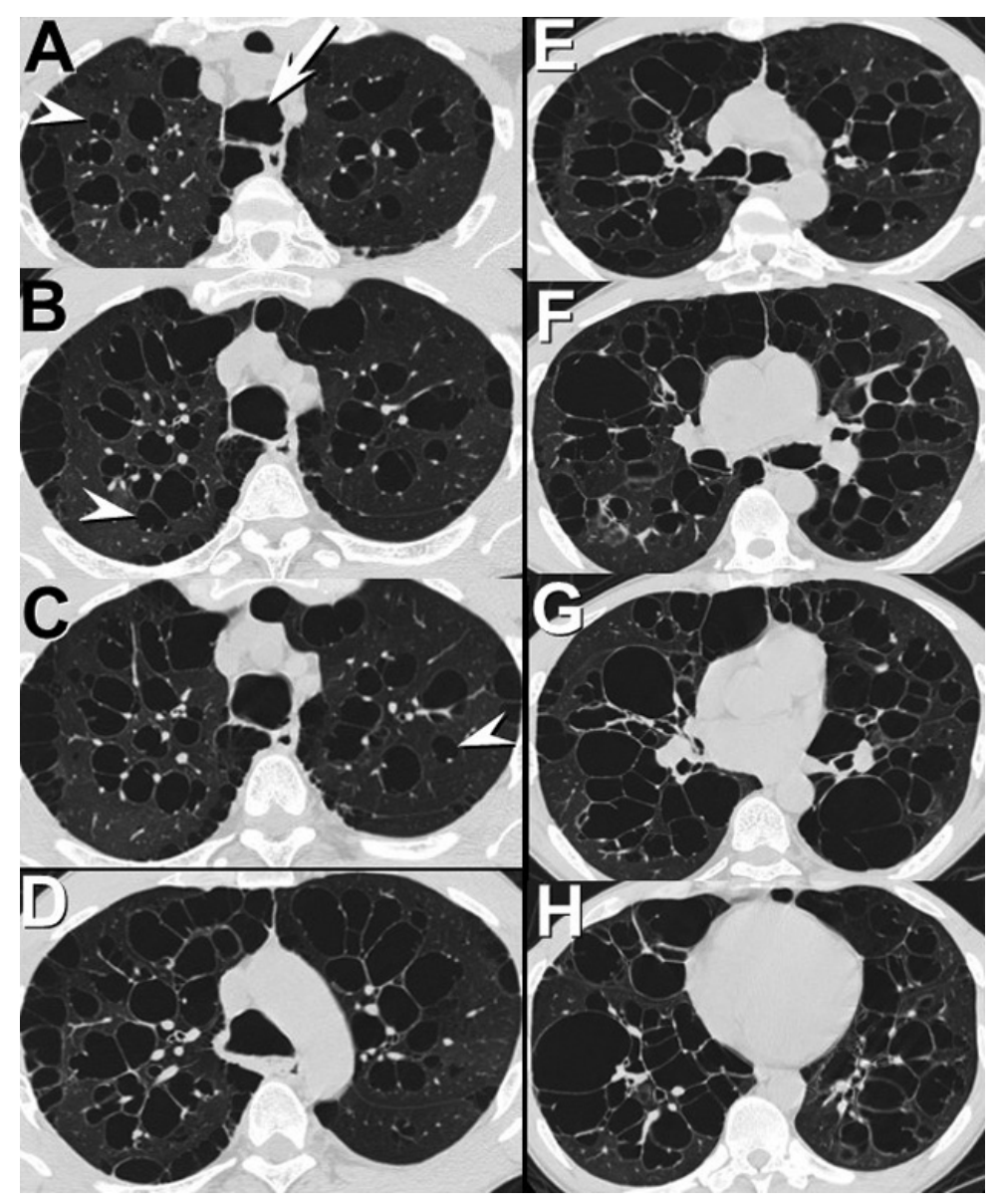

Figure 4. Axial thoracic CT images displayed in lung windows shows numerous, variably-sized thin-walled cystic lesions in a somewhat clustered distribution bilaterally.

Note the close relationship of the cystic lesions to adjacent pulmonary arteries (arrowheads); this relationship is consistent with the normal bronchoarterial relationship and identifies the cystic lesions as dilated bronchi. The trachea and mainstem bronchi show a mildly saccular appearance also. 
Additionally, centrilobular emphysema lacks a definable wall, whereas bronchiectasis and cystic lung disorders have definable, often thin, walls (Figure 5).

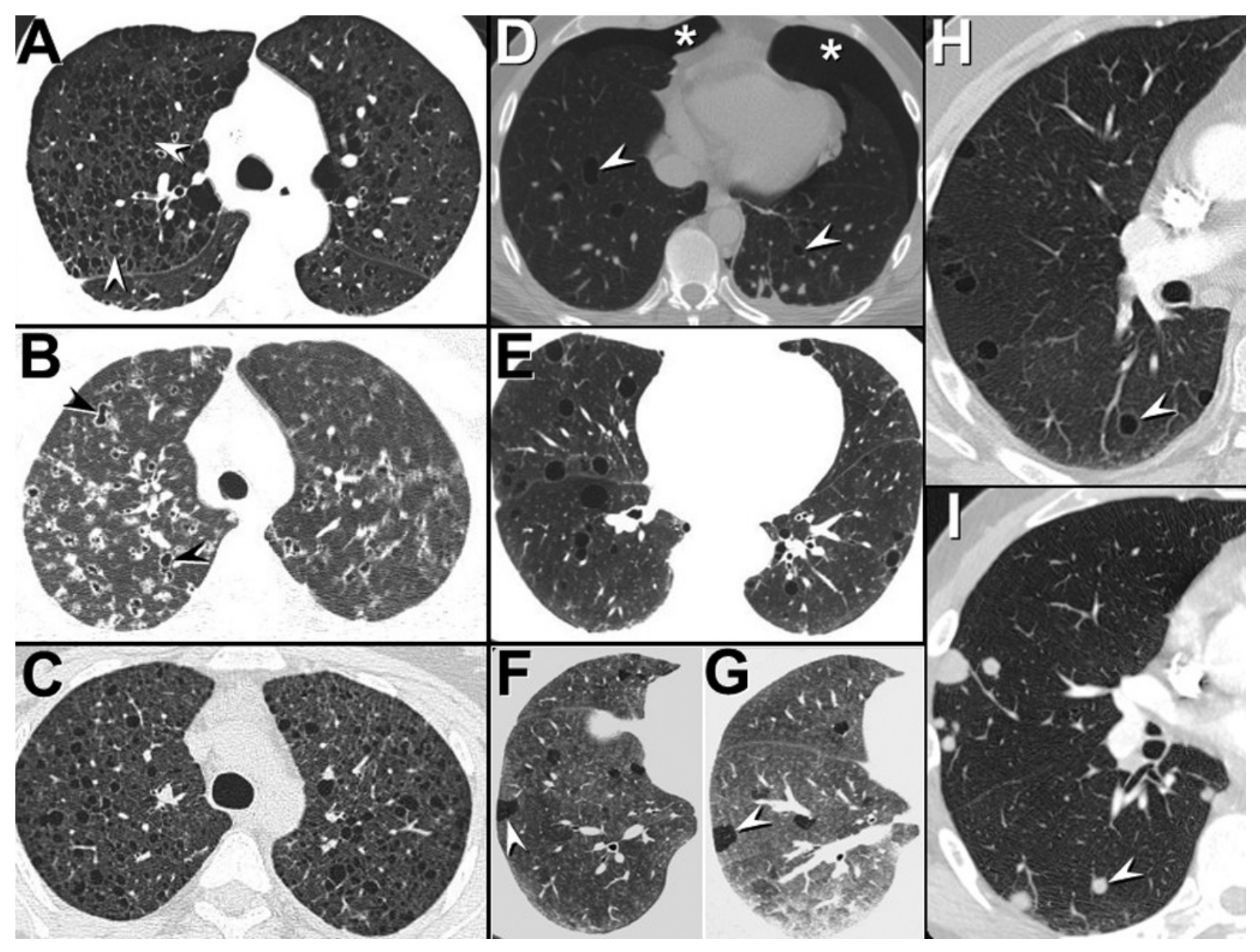

Figure 5. Various cystic pulmonary disorders. (A) Centrilobular emphysema. Note the lack of a definable wall surrounding the cystic lesions and the presence of the central "dot" within the area of low attenuation (arrowheads), representing the centrilobular artery. Occasionally this dot is not readily visible or may appear eccentrically located; the key to identification rests with reviewing multiple regions to identify the characteristic relationship. (B) Langerhans cell histiocytosis. The cysts in this disorder are typically distributed in the upper lobes and often display "bizarre" shapes (arrowheads), and associated pulmonary abnormalities, typically pulmonary nodules, are common.

Patients are usually smokers. (C) Lymphangioleiomyomatosis. The cysts in this disorder are more uniform in appearance, with normal intervening lung, and are distributed throughout the upper, mid, and lower lungs evenly, and no internal opacity is present. Spontaneous pneumothorax may occur. Patients are typically women of childbearing age or patients with tuberous sclerosis. (D) Birt-Hogg-Dubé syndrome. The cysts in this disorder (arrowheads) are more basally distributed and may be associated with spontaneous pneumothorax $\left(^{*}\right)$; affected patients often have renal and skin tumors as well. (E) Lymphocytic interstitial pneumonia. The cysts in this disorder vary in size and number, but may be associated with infiltrative pulmonary findings, such as ground- 
glass opacity, nodules, and interlobular septal thickening. The cysts typically do not

form clusters. Differentiation from other cystic pulmonary disorders, particularly lymphangioleiomyomatosis, can be difficult. ( $F$ and $G$ ) Lobular low attenuation due to small airway obstruction and air trapping. The low attenuation areas in patients with small airway obstruction producing lobular low attenuation typically do not show walls, and the low attenuation regions have a polygonal shape, reflecting the shape of the affected secondary pulmonary lobule (arrowheads). ( $\mathrm{H}$ and I) Cystic pulmonary metastases. Typically cystic pulmonary malignancies, whether primary or secondary, show some degree of wall thickness and irregularity. Occasionally cystic metastatic

disease may appear uniformly thin-walled (arrowheads, $\mathrm{H}$ ), as in this patient with metastatic testicular carcinoma. Often the clinical history will clarify the situation; the cystic appearance typically develops following treatment (occasionally some tumors,

such as metastatic angiosarcomas, may appear as thin-walled cysts even before therapy). However, serial scanning, or review of previous imaging (I), will usually resolve the issue. In this case, the previous imaging (I) shows solid metastatic nodules (arrowheads, I) where the cysts (arrowheads, H) subsequently developed. All the cysts on the follow up imaging in this patient could be correlated with metastatic nodules on the pre-treatment imaging.

Cystic pulmonary disorders may be distinguished from one another, with variable success, using patient demographics, the shape of the cysts, the presence / absence of associated pulmonary nodules and other pulmonary findings, the distribution of the cysts, and behavior of the lesions over time (Figures 5B-E). Lobular low attenuation due to small airway obstruction-induced air trapping typically produces polygonal-shaped areas of low attenuation which lack true walls; the polygonal shape reflects the shape of the affected secondary pulmonary lobule (Figure 5F and G). Cystic pulmonary metastatic disease usually results in cystic lesions that show some degree of wall thickness and irregularity, allowing them to be distinguished from other cystic pulmonary disorders. However, occasionally, the walls of cystic pulmonary metastases may show a uniformly thin-wall, closely simulating cystic pulmonary disease (Figure $5 \mathrm{H}$ ). In this setting, clinical history and the behavior of the cystic lesions over time usually clarifies the situation (Figure 5I). Bronchiectasis results in cystic lesions of variable size and wall thickness, but often the cysts have a clustered distribution, rather than the more dispersed appearance typical of other cystic pulmonary disorders. Furthermore, because bronchi and pulmonary arteries course adjacent to one another through the lung parenchyma, in the case of bronchiectasis, the cystic lesions are typically closely associated with an adjacent solid opacity, creating the appearance of a cyst with a "dot" along the cyst wall, as may be seen in this case (Figure 3).

What additional scan finding suggests the etiology of the bronchiectasis in this patient?

1. Associated fibrotic lung disease

2. High attenuation bronchial impaction

3. Numerous low air-fluid levels

4. The lower lobe distribution

5. Tracheobronchomegaly 


\section{Correct! \\ 5. Tracheobronchomegaly}

High attenuation bronchial impaction, often in the setting of central bronchiectasis, is very suggestive of allergic bronchopulmonary mycosis, but all the dilated bronchi in this case are clearly patent. No intrabronchial opacity or bronchial impaction is present, and no associated bronchiolitis is seen. Central bronchiectasis is frequently suggestive of allergic bronchopulmonary mycosis, whereas lower lobe predominant bronchiectasis is often seen with post-infectious etiologies. Bronchiectasis often favors the upper lobes in patients with cystic fibrosis, whereas middle lobe and lingua-predominant bronchiectasis is common with non-tuberculous mycobacterial infection and possibly even ciliary dyskinesia syndromes. The dilated bronchi in this patient are diffusely distributed throughout the upper, mid, and lower lungs; no particular zonal predilection is present. Numerous "low" air-fluid levels may be seen in various cases of bronchiectasis and may be useful for distinguishing bronchiectasis from other cystic pulmonary disorders, but this finding is not useful for distinguishing among the various causes of bronchiectasis. Fibrotic lung disease may produce traction bronchiectasis, but fibrotic abnormalities are lacking in this patient. However, the trachea and bilateral mainstem bronchi are abnormally enlarged, suggesting the diagnosis of tracheobronchomegaly, or MounierKuhn syndrome.

Diagnosis: Mounier-Kuhn syndrome (congenital tracheobronchomegaly)

\section{Discussion}

Congenital tracheobronchomegaly, or Mounier-Kuhn syndrome, is a rare disorder resulting from atrophy or even absence of elastic fibers and smooth muscle tissue within the airways, resulting in dilation of the trachea and proximal bronchi. Patients typically present in childhood or young adulthood, often with recurrent respiratory tract infections, although the present may vary from severe symptoms to asymptomatic detection, and the age range of presentation is wide. Eventually excessive airway collapsibility and severe airflow obstruction may result. Bronchiectasis is common, multiple airway diverticulae may be present, and emphysema may coexist. The diagnosis may be suggested at chest radiography in adult women when the tracheal transverse and sagittal diameters exceed $21 \mathrm{~mm}$ and $23 \mathrm{~mm}$, respectively, and when the mainstem bronchial transverse diameters exceed $19.8 \mathrm{~mm}$ and $17.4 \mathrm{~mm}$, respectively. In adult men tracheobronchomegaly may be diagnosed when the transverse and sagittal diameters of the trachea exceed $25 \mathrm{~mm}$ and $27 \mathrm{~mm}$, respectively, and when the transverse diameters of the right and left main bronchi exceed $21.1 \mathrm{~mm}$ and $18.4 \mathrm{~mm}$, respectively. At CT, adult tracheal, right mainstem, and left mainstem bronchial diameters exceeding $3 \mathrm{~cm}, 2.4 \mathrm{~cm}$, and / or $2.3 \mathrm{~cm}$, respectively, establish the diagnosis of tracheobronchomegaly. For simplicity, these numerical values are often used at radiography, although $\mathrm{CT}$ is preferred for diagnosis because the airway dimensions may be measured far more accurately than with radiography. The diagnosis of tracheobronchomegaly may also be made at bronchoscopy. 
Tracheobronchomegaly may also be encountered in the setting of fibrotic lung disease, but the mechanism in this circumstance is traction bronchiectasis, not an intrinsic tracheobronchial abnormality. Tracheobronchomegaly may also be seen in patients with connective tissue disorders, such as Ehlers-Danlos syndrome or children with cutis laxa.

\section{References}

1. Schwartz M, Rossoff L. Tracheobronchomegaly. Chest. 1994;106(5):1589-90. [CrossRef] [PubMed]

2. Smith DL, Withers N, Holloway B, Collins JV. Tracheobronchomegaly: an unusual presentation of a rare condition. Thorax. 1994;49(8): 840-1. [CrossRef] [PubMed]

3. Woodring J, Howard RS, 2nd, Rehm SR. Congenital tracheobronchomegaly (Mounier-Kuhn syndrome). J Thorac Imaging. 1999;6(1):1-10. [PubMed]

4. Shin MS, Jackson RM, Ho K-J. Tracheobronchomegaly (Mounier-Kuhn syndrome): CT diagnosis. AJR Am J Roentgenol. 1988;150:777-9. [CrossRef] [PubMed] 